\title{
Functional Dissociation between Anterior and Posterior Temporal Cortical Regions during Retrieval of Remote Memory
}

\author{
Takamitsu Watanabe, ${ }^{1 *}$ Hiroko M. Kimura, ${ }^{1 *}$ Satoshi Hirose,${ }^{1}$ Hiroyuki Wada, ${ }^{2}$ Yoshio Imai, ${ }^{2}$ Toru Machida, ${ }^{2}$ \\ Ichiro Shirouzu, ${ }^{2}$ Yasushi Miyashita, ${ }^{1}$ and Seiki Konishi ${ }^{1}$ \\ ${ }^{1}$ Department of Physiology, The University of Tokyo School of Medicine, 7-3-1 Hongo, Bunkyo-ku, Tokyo 113, Japan and 2Department of Radiology, NTT \\ Medical Center Tokyo, Tokyo 141, Japan
}

Retrieval of remote memory is considered to differentially involve the anterior and posterior temporal neocortices. Previous neuropsychological studies suggest that the different posterior temporal cortical regions are involved in the retrieval of remote memory of different categories of stimuli, whereas the anterior region is involved more generally in remote memory retrieval. In the present study, using functional magnetic resonance imaging of human brains, we tested this dissociation by examining the more precise characteristics of the anterior and posterior temporal cortical regions. Two categories of stimuli, faces and scenes, were used for paired stimuli to be retrieved, and the brain activity during retrieval of paired stimuli that were learned immediately before the scanning was compared with that during retrieval of paired stimuli that were learned $\sim 8$ weeks earlier. We found that the different posterior temporal cortical regions were activated during retrieval of different categories of remote memory in a category-specific manner, whereas the anterior temporal cortical region was activated during retrieval of remote memory in a category-general manner. Furthermore, by applying a multivariate pattern analysis to psychophysiological interactions during retrieval of remote memory relative to recent memory, we revealed the significant interaction from the category-specific posterior temporal cortical regions to the category-general anterior temporal region. These results suggest that the posterior temporal cortical regions are involved in representation and retrieval of category-specific remote memory, whereas the anterior cortical temporal region is involved in category-general retrieval process of remote memory.

\section{Introduction}

Neuropsychological and neuroimaging studies revealed that damage to the medial temporal lobe (MTL) including the hippocampus causes anterograde and temporally limited retrograde amnesia (Scoville and Milner, 1957; Warrington, 1975; ZolaMorgan and Squire, 1990; Kapur and Brooks, 1999; Manns et al., 2003), and that the MTL is involved in memory consolidation (Stark and Squire, 2000; Haist et al., 2001; Axmacher et al., 2008; Tambini et al., 2010). In contrast, neuropsychological studies revealed that lesions in the temporal neocortex induce severe and temporally extended retrograde amnesia (Hodges et al., 1992; Kapur et al., 1992; Damasio et al., 1996; Bayley et al., 2005), and

Received Nov. 4, 2011; revised April 5, 2012; accepted April 16, 2012.

Author contributions: T.W., H.M.K., Y.M., and S.K. designed research; T.W., H.M.K., S.H., H.W., Y.I., T.M., I.S., and S.K. performed research; T.W. contributed unpublished reagents/analytic tools; T.W. and S.K. analyzed data; T.W., Y.M., and S.K. wrote the paper.

This work was supported by a Grant-in-Aid for Specially Promoted Research (19002010) to Y.M., a Grant-in-Aid for Scientific Research B (22300134) to S.K., a grant from the Japan Society for the Promotion of Science Research Foundation for Young Scientists (222882) to T.W., and a research grant from Takeda Science Foundation to Y.M. We thank Yuriko Suzuki for technical assistance.

${ }^{*}$ T.W. and H.M.K. contributed equally to this work.

The author(s) declare(s) no competing financial interests.

Correspondence should be addressed to either T. Watanabe or S. Konishi, Department of Physiology, The University of Tokyo School of Medicine, 7-3-1 Hongo, Bunkyo-ku, Tokyo 113, Japan. E-mail: takawatanabe-tky@umin.ac.jp or konishi@m.u-tokyo.ac.jp.

DOI:10.1523/JNEUROSC1.5553-11.2012

Copyright $\odot 2012$ the authors $\quad 0270-6474 / 12 / 329659-12 \$ 15.00 / 0$ electrophysiological and neuroimaging studies demonstrated that the temporal neocortex contains neural representation of remote memory (Sakai and Miyashita, 1991; Higuchi and Miyashita, 1996; Messinger et al., 2001; Naya et al., 2003; Takashima et al., 2006; Yamashita et al., 2009). These functional dissociations between the hippocampus and temporal neocortex have been discussed in several models such as the standard memory consolidation theory (Squire and Bayley, 2007) and multiple trace theory (Moscovitch et al., 2006).

Inside the temporal neocortex, the anterior and posterior cortices are considered to be differentially involved in remote memory retrieval (Martin, 2007; Patterson et al., 2007). The posterior temporal cortex is considered to have a fine-grained structure related to object category (Martin, 2007). Neuropsychological studies reported that lesions in the posterior temporal cortex caused amnesia of a specific category of knowledge (Tranel and Damasio, 1985; Hart and Gordon, 1990; Martin et al., 1995; Damasio et al., 1996). For instance, the inferior temporal cortex was specifically related to semantic knowledge of nonliving things such as tools (Damasio et al., 1996; Martin et al., 1996). The more dorsal part of the temporal cortex is selectively related to knowledge of living things such as faces (Chao et al., 1999; Polyn et al., 2005).

In contrast, the anterior temporal lobe is considered to have more general roles in semantic remote memory processing (Gauthier et al., 1997; Martin and Chao, 2001; Patterson et al., 2007). 
Neuropsychological studies reported that lesions in the anterior region caused amnesia of broad categories of knowledge (Mummery et al., 2000; Chan et al., 2001; Desgranges et al., 2007). Other studies using semantic dementia patients commonly reported lesions in the anterior temporal lobe (Davies et al., 2004; Nestor et al., 2006). Neuroimaging studies also revealed that the anterior temporal regions are commonly activated during psychological tasks requiring various categories of semantic memory (Devlin et al., 2000; Bright et al., 2004; Rogers et al., 2006).

Together, as described in a "distributedplus-hub view" theory (Patterson et al., 2007), the previous literature suggests a hypothesis that the different posterior temporal regions are involved in retrieval of remote memory of different categories in a category-specific manner, whereas the anterior temporal cortex is involved in remote memory retrieval in a category-general manner. In the present functional magnetic resonance imaging (fMRI) study, we aim to examine the hypothesized functional difference between the anterior and posterior temporal regions during remote memory retrieval.

\section{Materials and Methods}

Overall experiment design. We adopted a task design where subjects separately learned two different categories of stimuli (faces and scenes) through a paired association (PA) task (Fig. 1 A) (Higuchi and Miyashita, 1996; Yamashita et al., 2009). Approximately 8 weeks after they learned the first set of stimuli (remote pairs), the subjects studied another new set of stimuli (recent pairs). Approximately $10 \mathrm{~min}$ later, they underwent the PA task in the fMRI scanner as a test of both the recent and remote pairs (Fig. $1 B$ ). By comparing the brain activity during retrieval of recent versus remote memory, we confirmed the functional dissociation by identifying the category-specific regions in the posterior temporal neocortex and the category-general region in the anterior temporal neocortex. By applying multivariate pattern analysis (MVPA) of psychophysiological interaction (PPI), we further characterized the directional relationship between the anterior and posterior temporal neocortices.

Subjects and fMRI acquisition. Forty-three healthy right-handed subjects (18 males, 25 females; aged 20-28 years) participated in the present experiments after written informed consent was obtained. The MRI scanning was conducted using a 3 T MRI scanner (Philips Achieva 3.0T X-series, Rel. 2.6). The T1-weighted structural images were obtained for anatomical reference (resolution $=0.81 \times 0.81 \times 1.20$ $\mathrm{mm}^{3}$ ). Functional imaging used gradient echo echo-planar sequences $\left(\mathrm{TR}=2 \mathrm{~s}, \mathrm{TE}=35 \mathrm{~ms}\right.$, flip angle $=85^{\circ}$, resolution $=4 \times 4 \times 4 \mathrm{~mm}^{3}, 32$ slices). The first five functional images of each run were discarded to allow for the effects of transient magnetic saturation. In an additional experiment, 15 healthy right-handed subjects ( 9 male, 6 females) participated after written informed consent was obtained $\left(\mathrm{TR}=3 \mathrm{~s}, \mathrm{TE}=50 \mathrm{~ms}\right.$, flip angle $=90^{\circ}$, resolution $4 \times 4 \times 4 \mathrm{~mm}^{3}, 1.5 \mathrm{~T}$ ). The whole procedure of the MRI scanning was approved by the institutional review board of The University of Tokyo School of Medicine.

Task and stimuli. Stimuli used in the present PA tasks consisted of monochrome face and scene images. Images of the pairs comprised the same category of images (i.e., a face and another face image; a scene and another scene image). The stimuli for recent pairs and those for remote pairs were counterbalanced across subjects. That is, the stimuli for the recent pairs in some subjects were used as those for the remote pairs in the other subjects.

The task design was similar to that used in our previous human fMRI study (Fig. 1A) (Yamashita et al., 2009). The PA task began with the presentation of one of the paired images as a cue stimulus (cue period), which was followed by the presentation of two images as a target and a distractor, which were located horizontally side by side (choice period). The subjects were instructed to choose one of the two images as an image paired to the cue stimulus. A trial in the test sessions comprised a cue period of $1 \mathrm{~s}$, a fixation gap of $0.5 \mathrm{~s}$, a choice period of $2 \mathrm{~s}$, and an intertrial interval of $0.5 \mathrm{~s}$ ( $4 \mathrm{~s}$ in total). Another version of the PA task used in the study sessions additionally had a feedback period after the choice period, where the subjects were visually indicated as to whether their answer was correct or not (correct: $\mathrm{o}$, incorrect: $\mathrm{x}$ ).

Independently of the PA tasks, we had the subjects undergo a functional localizer session for face and scene stimuli, because the perception regions have been suggested to interact with the hippocampus during memory formation (Takashima et al., 2009; Tambini et al., 2010). As in standard procedures of localization scans (Epstein and Kanwisher, 1998), the session consisted of pseudorandomly ordered 8 face blocks and 8 scene blocks, each of which contained 20 face and scene images, respectively. Each of the images was presented for $0.3 \mathrm{~s}$ with a fixation gap of $0.5 \mathrm{~s}$. The subjects were instructed to look at the images and press a button when the same image was successively presented.

Experiment schedule. The subjects underwent a study session for remote pairs (not scanned, $2 \mathrm{~d}$ ), a study session for recent pairs (not scanned), a test session (scanned), and a postscan session with confidence rating (Fig. $1 \mathrm{~B}$ ). The study session for the remote pairs comprised learning of 30 pairs of face stimuli and 30 pairs of scene stimuli (Round 1) and relearning of the same pairs (Round 2). Before Round 1, the subjects were presented with unfamiliar stimuli twice to familiarize them. The stimuli were confirmed to be unfamiliar to the subjects before the entire experiment, and when any one of the stimuli was reported to be familiar, the stimulus was replaced by a new stimulus. Round 2 was separated from Round 1 by $\sim 2$ weeks. Approximately 8 weeks after completion of Round 2 for the remote pairs, the subjects underwent the study session 
for the recent pairs. As in the study session for the remote pairs, they were first presented with unfamiliar images twice for familiarization and then learned a new set of 60 pairs of stimuli (30 pairs of face stimuli and 30 pairs of scene stimuli).

In the study session for the remote pairs, the subjects were required to learn the pairs until they could conduct the same PA trials three times successively with $100 \%$ accuracy, whereas, in the study session for the recent pairs, they were required to learn the pairs with $\sim 70 \%$ accuracy, which was previously shown to be successful in matching the performance in recent trials with that in remote trials during the scanned session (Yamashita et al., 2009). To achieve these criteria, the subjects needed to learn the recent pairs $2.2 \pm 0.7$ times (mean $\pm \mathrm{SD}$ ), whereas they needed to learn the remote pairs $27.5 \pm 8.2$ times.

Approximately $10 \mathrm{~min}$ after completing the study session for the recent pairs, the test session was administered while six runs of functional imaging were acquired. Each of the runs contained 20 PA trials: 10 for PA trials of the recent pairs and $10 \mathrm{PA}$ trials of the remote pairs. These PA trials (30 recent face, 30 recent scene, 30 remote face, and 30 remote scene during the six runs in total) and fixation trials were pseudorandomly presented for an eventrelated fMRI design. To select PA trials in which the subjects retrieved firm memory, we used a confidence rating that the subjects scored in the postscan PA task. The subjects were instructed to rate their confidence in each of their answers as low/middle/high when they estimated that the answer would be correct by $50 / 70 / 90 \%$ or more, respectively.

Behavioral analysis. To validate the confidence rating made in the postscan session, we estimated how accurately the confidence was rated by calculating the percentage of the correct trials in the postscan session for each of the low/middle/high confidence trials.

Because we adopted PA tasks that required responses in a forcedchoice manner, the subjects could select correct choices both inside and outside of the fMRI scanner with a probability of 0.25 by chance. To exclude such unreliable correct trials, we used the confidence rating as in previous studies (Takashima et al., 2009; Yamashita et al., 2009), and defined "remember trials" and "less-remember trials" in the scanned PA task. Remember trials, where successful retrieval was expected to take place with reasonably high probability, were defined as the trials to which the subjects responded correctly both in the scanned PA task and in the postscan PA task, and also rated high or middle confidence in the postscan PA task. Less-remember trials were defined as the rest of the trials. The definition was adopted to achieve the balanced number of trials between the remember trials and the less-remember trials, which maximized statistical power to detect the difference in PPIs between the two types of trials in the later PPI analysis. Using the definition, we applied the repeated-measures two-way ANOVA (recent/remote $\times$ face/scene) to the response time for the remember trials and the retrieval accuracy.

fMRI analysis. Imaging data were analyzed using SPM2 (http://www. fil.ion.ucl.ac.uk/spm/). Functional images were realigned, slice timing corrected, normalized to the default template with interpolation to a $2 \times$ $2 \times 2 \mathrm{~mm}^{3}$ space, and spatially smoothed (full-width at half-maximum $=10 \mathrm{~mm}$, Gaussian filter). High-pass temporal filtering with a cutoff of $64 \mathrm{~s}$ was applied. In a single subject level, together with runspecific effects and other events of no interest, event timings of the eight types of trials (remember/less-remember $\times$ recent/remote $\times$ face/scene) were coded into a general linear model (GLM) using the canonical hemodynamic response function in SPM2, time-locked to the onset of the cue stimulus presentation.

The group analysis adopted a random effect model. To estimate the brain regions involved in retrieval of category-general recent/remote memory, we estimated the main effect of the recent/remote trials in the repeated-measures two-way ANOVA (recent/remote $\times$ face/scene). For the estimation of the regions related to the retrieval of category-general remote memory, we used the contrast images for (remote remember trials of the face + scene stimuli) $>$ (recent remember trials of the face + scene stimuli), whereas the opposite contrast images were used for the estimation of brain regions involved in the retrieval of category-general recent memory. Significant activations were detected using the threshold of $p<0.05$ corrected for a small volume around the coordinates reported in our previous study (Yamashita et al., 2009). We then estimated brain regions that were involved in category-specific remote memory by calculating the interaction in the repeated-measures two-way ANOVA of the brain activity. To estimate the brain regions related to retrieval of facespecific remote memory we contrasted images for (remote remember trials of the face - scene stimuli) $>$ (recent remember trials of the face scene stimuli). To estimate the brain activity related to the retrieval of scene-specific remote memory, we used the contrast images for (remote remember trials of the scene - face stimuli) $>$ (recent remember trials of the scene - face stimuli). Significant activations were detected using the threshold of $p<0.05$, familywise error (FWE)-corrected in the gray matter in the temporal lobe (Tzourio-Mazoyer et al., 2002).

For each of the temporal regions detected by using the above ANOVA contrasts, we examined the Pearson's correlation coefficients between the brain activity and one of the following three different behavioral scores. First, we used the proportion of the remember trials as the behavioral score. Second, we used the "confidence ratio" that reflected how confidently each subject responded in correct trials on average. The confidence ratio was defined as the proportion of the number of remember trials with only high confidence to that of all of the remember trials with high or middle confidence. That is, the subjects with greater confidence ratio are considered to have firmer memory. Third, to exclude a concern that the confidence rating was biased by the subjects' individual traits on their self-evaluation, we used the discrepancy of the confidence rating from the actual performance. The discrepancy was defined as the difference between the number of correct trials expected from the confidence rating and the actual number of correct trials in the postscan PA task (i.e., the expected number $=0.5 \times$ the number of low confidence trials in the postscan PA task $+0.7 \times$ the number of middle confidence trials $+0.9 \times$ the number of high confidence trials). That is, the subjects with a large discrepancy overestimated their own accuracy in the PA task.

Imaging data collected during the functional localizer session were preprocessed in the same manner as the above analyses. In a single-level analysis, two regressors for the face and scene trials were modeled in a GLM. In a group-level analysis using a random effect model, the contrast images for the face minus scene stimuli were used for the detection of the fusiform face area (FFA) in the fusiform gyrus, whereas the contrast images for the scene minus face stimuli were used for the detection of the parahippocampal place area (PPA) in the parahippocampal gyrus (Kanwisher et al., 1997; Haxby et al., 2001) $(p<0.05$, FWE-corrected in the temporal lobe) (Tzourio-Mazoyer et al., 2002).

PPI estimation and MVPA. PPI (Friston et al., 1997; Etkin et al., 2006; Stephan and Friston, 2010; Edelson et al., 2011) was estimated among the brain regions detected by the ANOVA contrasts and the localizer scans: the brain region related to retrieval of the category-general recent memory (the hippocampal region), that related to retrieval of the categorygeneral remote memory (the anterior temporal region), that related to retrieval of the category-specific remote memory (the posterior temporal regions), and that related to category-specific perception (the FFA/PPA). The method of estimating PPI was based on the implementation in SPM2 (Friston et al., 1997). In a single-subject level, PPI for recent/remote trials was calculated between the recent/remote trials versus fixation trials (Friston et al., 1997; Etkin et al., 2006). We conducted this estimation for each category of stimuli and obtained four sets (i.e., the recent/remote $X$ face/scene trials) of 12 PPIs (i.e., selections of two regions of all the four regions, except for the auto-recursive cases, with the direction of the PPI taken into consideration) for each of the subjects (see Fig. 5A).

To compare the PPIs ( $z$ values) for each of the 12 region pairs, we applied paired $t$ tests across subjects to the PPIs for the recent trials and the remote trials. However, no PPI exhibited significant difference on its own (Table 1). Therefore, we next attempted to search for a combination of PPIs, rather than a single PPI, that exhibited the largest difference in magnitude between the recent and remote trials as follows. First, based on the absolute difference in each PPI value between the recent and remote trials (Table 1), we ranked all of the 12 PPIs in descending order according to the obtained absolute $t$ values (Fig. 5A, Table 1) (Mitchell et al., 2004; De Martino et al., 2008; Dosenbach et al., 2010). Second, we sequentially built 12 candidate sets of the multiple PPIs on the basis of the PPI ranking (Table 1), such as only \#1, a set of \#1 and \#2, a set of \#1, \#2, and $\# 3, \ldots$ and a set of all the 12 PPI (Fig. 5B). 
Table 1. Brain activations related to retrieval of recent/remote memory

\begin{tabular}{|c|c|c|c|c|c|}
\hline \multirow[b]{2}{*}{$R / L$} & \multirow[b]{2}{*}{$\begin{array}{l}\text { Anatomical } \\
\text { label }\end{array}$} & \multicolumn{3}{|c|}{ MNI coordinates } & \multirow[b]{2}{*}{$t$ value } \\
\hline & & $x$ & $y$ & $z$ & \\
\hline \multicolumn{6}{|l|}{$\begin{array}{l}\text { Temporal lobe } \\
\text { remote }>\text { recent }\end{array}$} \\
\hline$\stackrel{\mathrm{L}}{\text { recent }>\text { remote }}$ & Ant temp & -30 & 6 & -40 & 4.0 \\
\hline $\begin{array}{c}\mathrm{R} \\
(\text { face }>\text { scene }) \times(\text { remote }>\text { recent })\end{array}$ & Hippo & 30 & -38 & -4 & 3.5 \\
\hline $\begin{array}{l}\mathrm{R} \\
(\text { scene }>\text { face }) \times(\text { remote }>\text { recent })\end{array}$ & STS & 46 & -50 & 20 & 4.8 \\
\hline $\mathrm{R}$ & ITG & 42 & -50 & -8 & 4.3 \\
\hline \multicolumn{6}{|l|}{$\begin{array}{l}\text { Others } \\
\quad \text { remote }>\text { recent }\end{array}$} \\
\hline $\mathrm{R}$ & IFG & 56 & 34 & 14 & 6.7 \\
\hline$R$ & IFG & 64 & 28 & 26 & 5.8 \\
\hline L & IFG & -54 & 38 & 12 & 5.6 \\
\hline \multicolumn{6}{|l|}{ recent $>$ remote } \\
\hline$R$ & SFG & 34 & 46 & 34 & 6.0 \\
\hline$R$ & SFG & 40 & 56 & 12 & 5.8 \\
\hline$R$ & MFG & 38 & 46 & 20 & 6.2 \\
\hline R & Precuneus & 12 & -70 & 38 & 9.8 \\
\hline L & IPL & -40 & -68 & 36 & 5.6 \\
\hline R & IPL & 38 & -48 & 36 & 5.6 \\
\hline
\end{tabular}

Ant temp, anterior temporal region; IFG, inferior frontal gyrus; SFG, superior frontal gyrus; MFG, middle frontal gyrus; IPL, inferior parietal lobe; Hippo, hippocampus; STS, superior temporal sulcus; ITG, inferior temporal gyrus. R, Right; L, left.

Third, for each of the 12 candidate PPI sets, we determined how accurately a multivariate classifier function predicted whether the set of multiple PPIs was attributed to the recent trials or the remote trials. The MVPA used a linear support vector machine (SVM) implemented in LIBSVM (www.csie.ntu.edu.tw/ cjlin/libsvm/) (Kamitani and Tong, 2005; Hassabis et al., 2009; Dosenbach et al., 2010; Watanabe et al., 2011). To estimate the classification accuracy of the trained classifier function, we used a leave-one-out cross-validation (Kamitani and Tong, 2005; Dosenbach et al., 2010) and the statistical significance for the accuracy was evaluated by a permutation test (Hassabis et al., 2009; Dosenbach et al., 2010; Watanabe et al., 2011) (Fig. 5C). To avoid a circular analysis (Kriegeskorte et al., 2009), the PPI data used in the $t$ test-based ranking were designed to be independent of the data used in tests of the classifier function in the MVPA. Consequently, following the above procedures, the PPI set that was estimated to exhibit the largest difference between the recent and remote trials was chosen as the set of significant PPIs. This combination of the $t$ test-based ranking (Mitchell et al., 2004; De Martino et al., 2008; Dosenbach et al., 2010) and MVPA (Xiong et al., 2001; Inza et al., 2004) has been validated as an appropriate method to select the most differential PPI set even when we do not have a priori information about how many PPIs are included in the most differential PPI set (Xiong et al., 2001; Inza et al., 2004; Ruiz et al., 2006; Saeys et al., 2007).

This newly developed MVPA to PPI data among the temporal regions might not be automatically applied to the investigation of the PPI network in the whole brain. When we investigated the PPI network within the temporal lobe, there were potentially $2^{12}-1=4095$ patterns of PPI networks among the four regions of interest (i.e., there were $4 \cdot 3=12$ PPIs among four regions). Using the univariate filter method (i.e., ranking based on $t$ values; Table 2), we selected and investigated 12 of the 4095 networks. In contrast, if we add the frontal and parietal regions to the regions of interest (ROIs), the number of potential networks will explosively increase. As shown in Table 1, there are nine frontal and parietal regions related to retrieval of recent/remote memory, and, thus, the number of ROIs will be 12 , which means that the number of the potential networks will be $2^{132}-1=5.4 \times 10^{39}$ (i.e., there were $12 \times 11=132$ PPIs). Using the univariate filter method, 132 patterns will be selected from the $5.4 \times 10^{39}$ potential networks. In such a situation, it would be too difficult to avoid false negative and detect valid results. Considering this technical limitation, we focused on the neural mechanism in the temporal lobe in the present study.
Additional experiment. We conducted an additional experiment to examine whether different amounts of repetition of training had significant effects on the brain activity in the regions that were found in the above main experiment. Fifteen subjects participated in this experiment, and underwent PA tasks using 40 face-face and 40 scene-scene paired stimuli. Ten face pairs and 10 scene pairs were learned 28 times, while the others (30 face pairs and 30 scene pairs) were learned 3 times. Approximately 10 min after the training, the subjects underwent a PA task in the fMRI scanner as a test of both the paired stimuli following the heavy and light repetition of training. As in the main experiment, after the fMRI scanning, the subjects were instructed to rate their confidence in each of their answers as low/middle/high when they estimated that the answer would be correct by $50 / 70 / 90 \%$ or more, respectively. In this additional experiment, we focused on trials during which the subjects correctly retrieved both in the fMRI scanning and the confidence rating test and rated high confidence. By focusing on this type of trial, the number of correct trials with high confidence was balanced between the correct trials following the heavy repetition and those following the light repetition.

\section{Results}

\section{Behavioral results}

We first evaluated the accuracy of the confidence rating taken during the postscan session. The percentage of correct trials for the recent face pairs with low/middle/high confidence was $52 \pm$ $3 / 69 \pm 2 / 91 \pm 2 \%$, whereas that for the recent scene pairs was $46 \pm 3 / 70 \pm 1 / 89 \pm 3 \%$, respectively (Fig. $2 A$ ). The percentage of correct trials for the remote face pairs with low/middle/high confidence was $51 \pm 2 / 72 \pm 3 / 92 \pm 2 \%$ (mean \pm SEM), whereas that for the remote scene pairs was $48 \pm 2 / 71 \pm 1 / 91 \pm 3 \%$, respectively (Fig. $2 \mathrm{~A}$ ). These results confirm that the confidence rating is expected to accurately reflect the degree of successful retrieval in individual trials in the PA task.

Using the validated confidence rating, we defined the remember and less-remember trials to analyze trials where successful retrieval was expected to take place (see Materials and Methods). The repeated-measures two-way ANOVA (recent/remote $\times$ face/scene) of the percentage of the remember trials showed neither significant main effect $(p>0.12)$ nor interaction $(p>0.48$; Fig. $2 B$ ). The same manner of analyses on the response time for the remember trials detected neither significant main effect $(p>$ 0.49 ) nor interaction ( $p>0.54$; Fig. $2 C$ ). These results suggest that, even after the long interval of 8 weeks, the behavioral performance in the PA task was successfully kept balanced among the four types of pairs, which is considered to be achieved by the different criteria in the training periods between the recent and remote stimuli.

\section{Brain regions related to retrieval of recent and remote memory}

We first evaluated the main effect of the recent versus remote trials in the repeated-measures two-way ANOVA (recent/remote $\times$ face/scene) (Fig. 3, Table 1). We found a right hippocampal region that exhibited a significantly main effect of recent $>$ remote $\left(F_{(1,42)}=10.5, p<0.05\right.$ in a small volume correction (SVC); Fig. $3 A$, Table 1). The hippocampal region did not show a differential activity between the recent face trials and recent scene trials $(p>0.7)$. We also estimated the main effect of remote $>$ recent, and found a left anterior temporal region $\left(F_{(1,42)}=16.0\right.$, $p<0.05$ in SVC; Fig. 3B, Table 1). The anterior temporal region did not exhibit a significantly different activity between the remote face trials and remote scene trials $(p>0.6)$. In a wholebrain analysis, the bilateral inferior frontal gyri showed significantly stronger activity during remote trials, whereas the right superior and middle frontal gyri, precuneus, and bilateral 
Table 2. Ranking of the difference in PPIs between the recent and remote trials

\begin{tabular}{|c|c|c|c|c|c|}
\hline \multirow[b]{2}{*}{ Rank } & \multirow[b]{2}{*}{ Interaction } & & \multicolumn{2}{|c|}{ Magnitude of PPI ( $z$ value, mean \pm SEM) } & \multirow{2}{*}{$\begin{array}{l}\text { Absolute value of } t \text { value in paired } t \text { test between } \\
\mathrm{PPI} \text { (remote) versus PPI (recent) }\end{array}$} \\
\hline & & & Remote trials & Recent trials & \\
\hline \multicolumn{6}{|c|}{ Face trials } \\
\hline \#3 & FFA to hippo & & $0.31 \pm 0.25$ & $0.24 \pm 0.28$ & 0.90 \\
\hline$\# 4$ & Ant temp to hippo & & $0.22 \pm 0.21$ & $0.26 \pm 0.26$ & 0.75 \\
\hline$\# 5$ & FFA to post temp (face) & & $-0.01 \pm 0.11$ & $0.02 \pm 0.12$ & 0.69 \\
\hline \#8 & Ant temp to FFA & * & $0.14 \pm 0.20$ & $0.17 \pm 0.25$ & 0.63 \\
\hline$\# 9$ & Post temp (face) to hippo & * & $0.35 \pm 0.20$ & $0.32 \pm 0.23$ & 0.63 \\
\hline$\# 10$ & Ant temp to post temp (face) & * & $-0.10 \pm 0.22$ & $-0.07 \pm 0.25$ & 0.63 \\
\hline \#11 & FFA to ant temp & & $0.17 \pm 0.25$ & $0.21 \pm 0.28$ & 0.59 \\
\hline$\# 12$ & Post temp (face) to FFA & & $0.15 \pm 0.12$ & $0.16 \pm 0.11$ & 0.55 \\
\hline \multicolumn{6}{|c|}{ Scene trials } \\
\hline$\# 5$ & Ant temp to hippo & & $0.23 \pm 0.26$ & $0.21 \pm 0.20$ & 0.78 \\
\hline$\# 6$ & Hippo to PPA & & $0.26 \pm 0.22$ & $0.29 \pm 0.25$ & 0.77 \\
\hline \#7 & PPA to post temp (scene) & & $0.19 \pm 0.29$ & $0.16 \pm 0.23$ & 0.75 \\
\hline \#8 & PPA to ant temp & * & $-0.17 \pm 0.30$ & $-0.19 \pm 0.22$ & 0.65 \\
\hline$\# 9$ & Post temp (scene) to hippo & * & $0.16 \pm 0.34$ & $0.19 \pm 0.38$ & 0.63 \\
\hline$\# 10$ & Ant temp to post temp (scene) & * & $0.21 \pm 0.38$ & $0.18 \pm 0.31$ & 0.60 \\
\hline \#11 & Ant temp to PPA & & $-0.17 \pm 0.24$ & $-0.19 \pm 0.25$ & 0.56 \\
\hline$\# 12$ & Post temp (scene) to PPA & & $-0.12 \pm 0.12$ & $-0.12 \pm 0.11$ & 0.50 \\
\hline
\end{tabular}

Post temp, posterior temporal region; FFA, fusiform face area; PPA, parahippocampal place area; Hippo, hippocampus; PPI, psychophysiological interaction. * ${ }^{*}$ ranking among the marked PPIs varied in different samples of the subjects (see Materials and Methods).
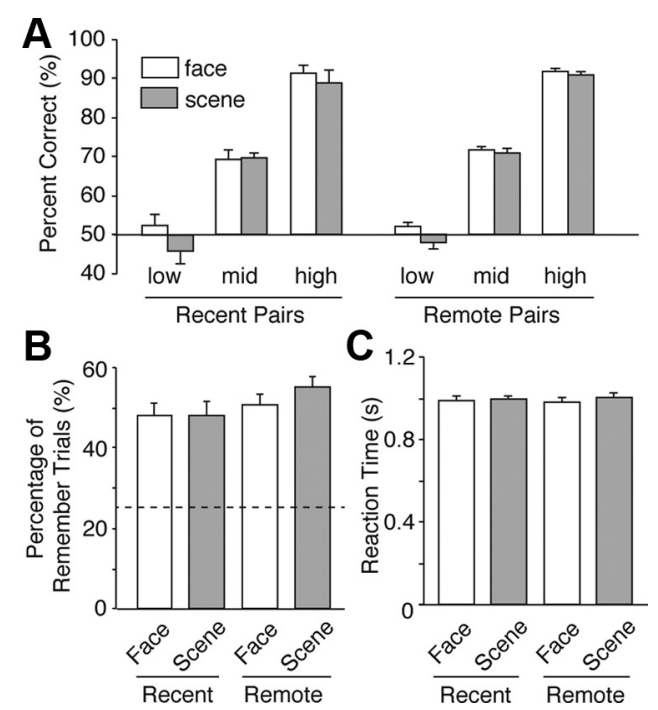

Figure 2. Behavioral results. $A$, Validation of confidence rating. The confidence rating in the postscan PA task reflected the degree of successful retrieval. In both the face and scene trials, trials with high confidence were correct by $\sim 90 \%$, those with middle confidence were correct by $\sim 70 \%$, and those with low confidence were correct by $\sim 50 \%$. Error bars indicate SEM. $\boldsymbol{B}$, Proportion of remember trials. There was no significant difference among the percentages of the remember trials in the recent/remote $\times$ face/scene trials (main effects: $p>0.12$; interaction: $p>0.48)$. The dashed line shows the proportion of the remember trials when the subjects selected answers by chance both in the test session and postscan session. $C$, Reaction time in remember trials. There was no significant difference in reaction time among the remember trials of the recent/remote $\times$ face/scene trials (main effects: $p>0.49$; interaction: $p>0.54$ ).

inferior parietal lobe showed larger activity during the recent trials $(p<0.05$, FWE-corrected; Table 1$)$. These results show that the hippocampal region is differentially involved in retrieval of category-general recent memory, whereas the anterior temporal region is differentially involved in retrieval of category-general remote memory.

We next searched for the brain activations that were related to retrieval of category-specific remote memory by estimating the effects of interaction between the recent/remote trials and face/ scene trials in the repeated-measures two-way ANOVA. We found that a region in the right superior temporal sulcus (STS) showed a significant interaction of (face $>$ scene) $\times$ (remote $>$ recent) $\left(F_{(1,42)}=23.0, p<0.05\right.$, FWE-corrected in the gray matter in the temporal lobe; Fig. $3 C$, Table 1). We also detected a region in the right inferior temporal gyrus (ITG) showing a significant interaction of $($ scene $>$ face $) \times$ (remote $>$ recent $)$ $\left(F_{(1,42)}=18.5, p<0.05\right.$, FWE-corrected in the gray matter in the temporal lobe; Fig. $3 D$, Table 1). In a whole-brain analysis, there was no significant effect of the interaction $(p>0.05$, FWEcorrected). These results suggest that the two posterior temporal regions were differentially related to retrieval of face- or scenespecific remote memory.

We then estimated the Pearson's correlation coefficients between the brain activity in these four regions (i.e., the hippocampus, anterior temporal region, and two category-specific posterior temporal regions) and the retrieval performance. In all four regions, the brain activity was significantly correlated with the proportion of the corresponding remember trials $(p<0.05 ;$ Fig. $4 A)$. As a control, we then estimated the correlation coefficients between the brain activity and the retrieval performance for the counterpart memory along the recent/remote axis (e.g., the activity in the hippocampal region related to recent memory was compared with the retrieval performance of the remote trials, rather than that of the recent trials). We did not find significant correlation for any of the four brain regions $(p>0.05)$, though there was no significant difference between the two types of correlation coefficients (e.g., correlation between the hippocampal activity and retrieval performance for the recent stim- 


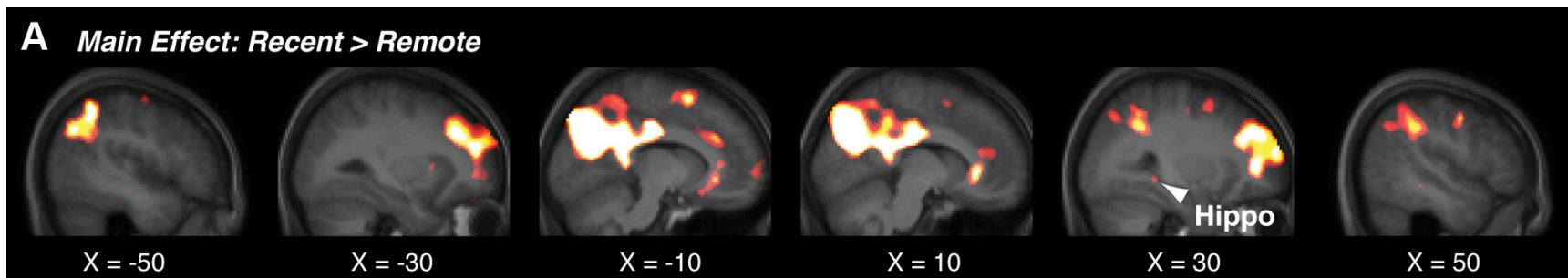

B Main Effect: Remote $>$ Recent

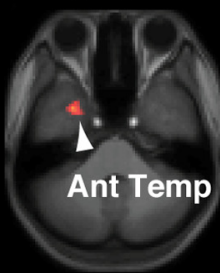

$Z=-40$

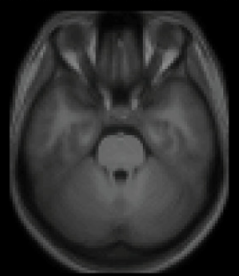

$Z=-30$

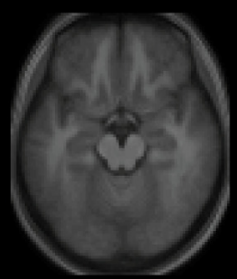

$Z=-20$

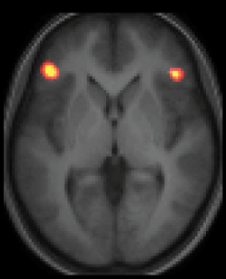

$Z=-10$

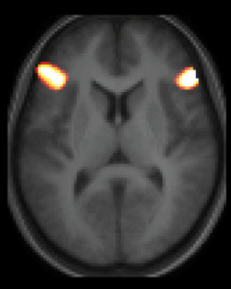

$Z=0$

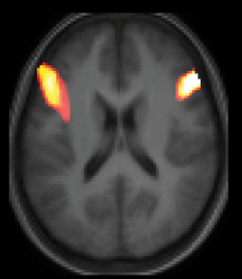

$Z=10$

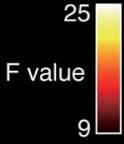

C Interaction: (Face $>$ Scene) $\times$ (Remote $>$ Recent)
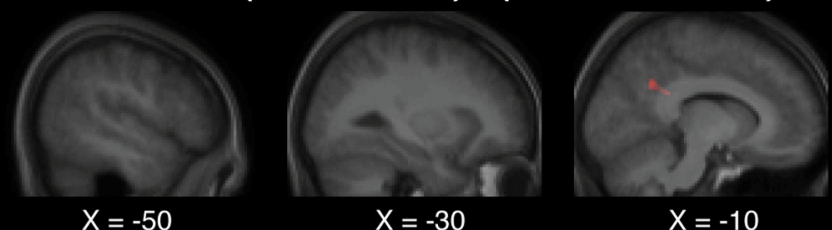

$X=-10$

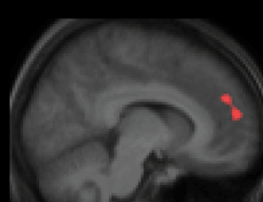

$X=10$

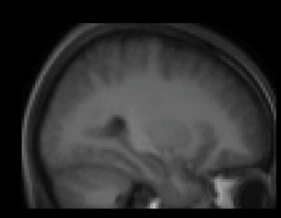

$\mathrm{X}=30$

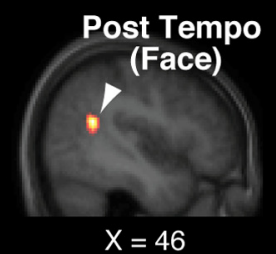

D Interaction: (Scene $>$ Face) $\times$ (Remote $>$ Recent)
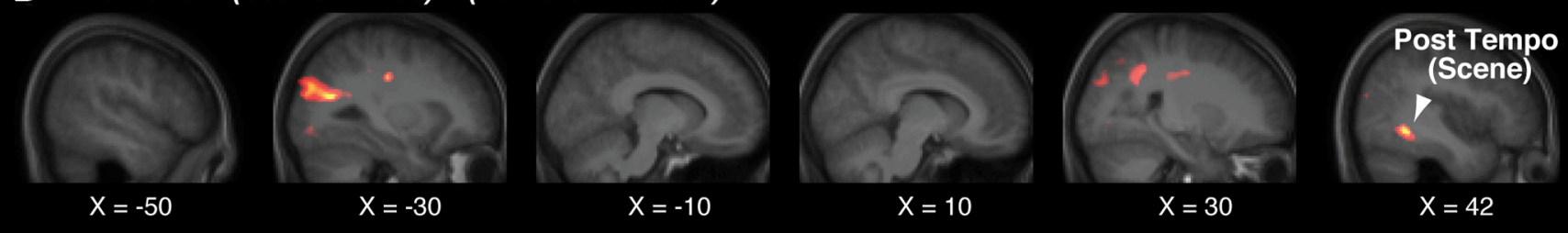

Figure 3. Brain regions related to retrieval of recent/remote memory. $A$, In the temporal lobe, the hippocampal region was activated during category-general recent memory (main effect of recent vs remote). $\boldsymbol{B}$, The anterior temporal region was activated during retrieval of category-general remote memory (main effect of remote and recent). $\boldsymbol{C}, \boldsymbol{D}$, The two different posterior temporal regions were involved in retrieval of category-specific remote memory (interaction of remote/recent $\times$ face/scene). Statistical significance is indicated using the color scale. Hippo, hippocampal region; Ant temperature, anterior temporal region; Post tempo, posterior temporal region.

uli vs correlation between the hippocampal activity and retrieval performance for the remote stimuli; $p>0.05)$.

By quantifying the proportional strength of the confidence as confidence ratio (see Materials and Methods), we also estimated the correlation between subjects' confidence ratio and brain activity in these four regions. In all four activated regions, the fMRI signal showed significantly positive correlation with the corresponding confidence ratio across subjects $(p<0.05 ;$ Fig. $4 B)$. We then estimated the correlation coefficients between the brain activity and the confidence ratio for the counterpart memory. We did not find any significant correlation for any of the four brain regions $(p>0.05)$, though there was no significant difference between the two types of correlations $(p>0.05)$. Moreover, to exclude a concern that subjects' traits as shown by their confidence rating would affect the significant correlation, we calculated correlations between the brain activity and the discrepancy of the confidence rating from the actual performance and found no significant result $(p>0.47)$. These correlation results indicate that the four temporal regions are related to successful retrieval of the corresponding long-term memory.

Comparisons of the brain activity between remember and less-remember trials gave further validation to the functional dissociation among these temporal regions (Fig. $4 C$ ). In the recent trials, the hippocampal region exhibited larger activity in the remember trials than in the less-remember trials in a categorygeneral manner $\left(t_{(42)}=2.1, p<0.05\right.$, in a paired $t$ test). In the remote trials, the hippocampal region showed significantly larger activity in the less-remember trials than in the remember trials $\left(t_{(42)}=2.1, p<0.05\right)$. The anterior temporal region showed larger activity in the remote remember trials than in the remote less-remember trials in a category-general manner $\left(t_{(42)}=2.2\right.$, $p<0.03)$. The category-specific posterior temporal region exhibited the same pattern in a category-specific manner (for face stimuli: $t_{(42)}=2.2, p<0.05$; for scene stimuli: $t_{(42)}=2.8, p<0.01$ ).

For further investigation of the functional dissociations, we also identified category-specific perception regions using a conventional functional localizer task (Kanwisher et al., 1997), because previous studies suggested that the perception regions are also involved in processing of remote memory (Takashima et al., 2009; Tambini et al., 2010). The FFA and PPA were detected in the temporal cortex as face- and scene-specific areas, respectively $\left(\right.$ FFA: $t_{(42)}=6.0,[42,-46,-22]$; PPA: $t_{(42)}=15,[30,-46$, $-14] ; p<0.05$, FWE-corrected in the temporal lobe). These activations were consistently close to those reported previously (Kanwisher et al., 1997; Haxby et al., 2001). 
A

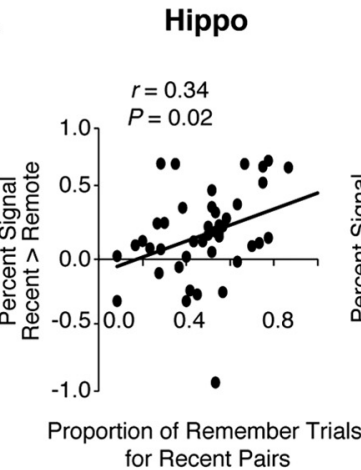

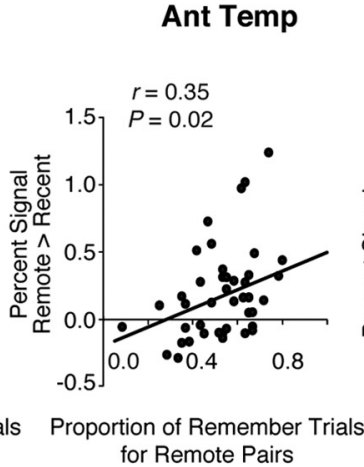

for Remote Pairs
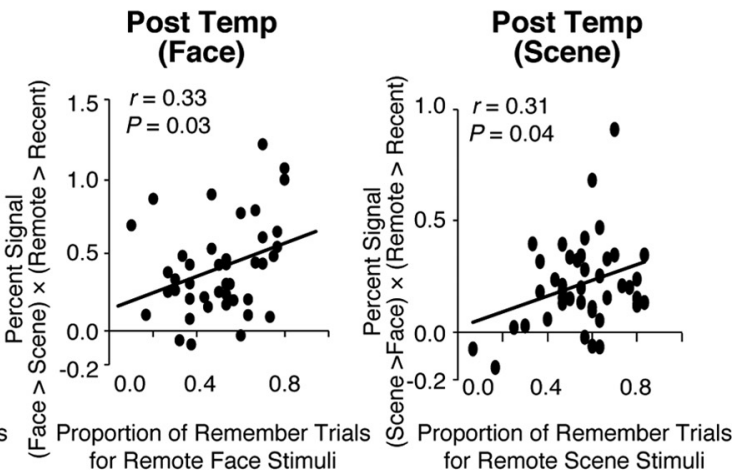

Post Temp

(Scene)
B Hippo

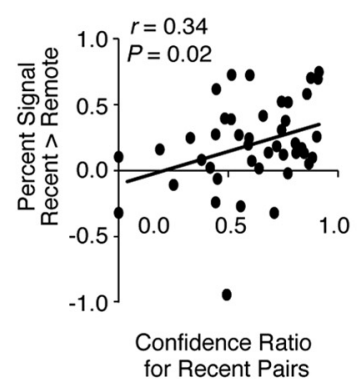

Ant Temp
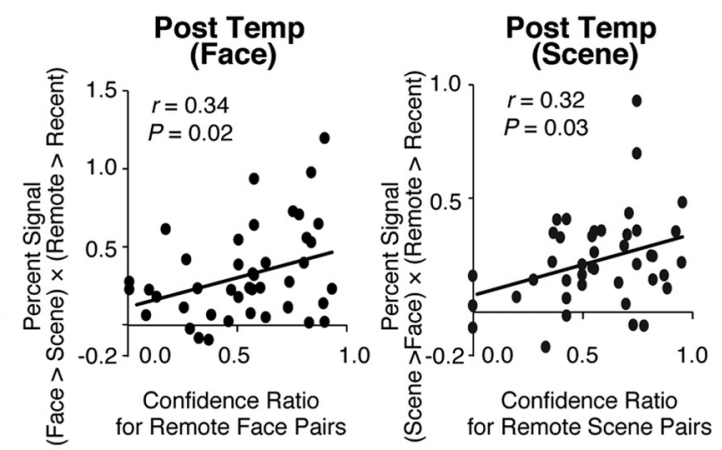

C
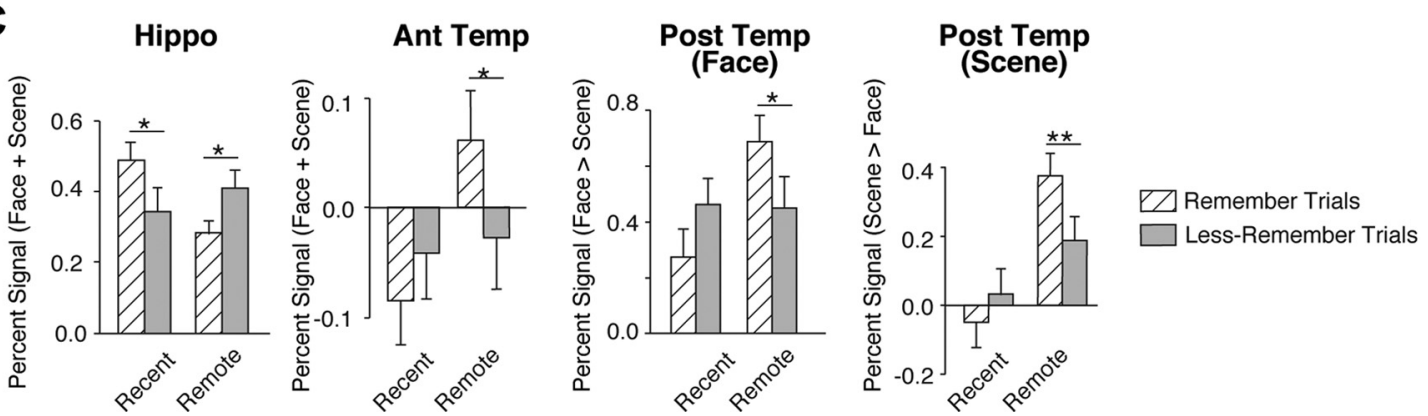

Figure 4. Characterization of the activated brain regions. $A$, Correlation with the proportion of remember trials. The brain activity in the four regions showed a significant positive correlation with the proportion of corresponding remember trials. The $x$-axis shows the proportion of remember trials (i.e., "the number of remember trials"/"the number of total trials"), and the $y$-axis shows the brain activity displayed in Figure 3. One plot represents data from one subject. $B$, Correlation with confidence ratio. The brain activity in the four regions showed a significant positive correlation with how firmly the subjects had confidence in their answers. The $x$-axis shows the confidence ratio (see text), and the $y$-axis shows the brain activity displayed in Figure 3 . One plot represents data from one subject. C, Comparison of percent signal between remember and less-remember trials. The $y$-axis shows the brain activity displayed in Figure 3 . Error bars indicate SEM. ${ }^{*} p<0.05 ;{ }^{* *} p<0.01$.

\section{MVPA of multiple PPI}

We then attempted to elucidate further functional difference between the anterior and posterior temporal cortical regions by comparing the interactions in the temporal lobe during retrieval of remote memory and those during retrieval of recent memory. In what follows, we separately conducted analyses for each category of stimuli. We first estimated PPIs among the following four regions for each category of stimuli: for the face trials, the hippocampal region, anterior temporal region, face-specific posterior temporal region, and FFA; for the scene trials, the hippocampal region, anterior temporal region, scene-specific posterior temporal region, and PPA. Figure $5 A$ presents representative PPI data in one subject consisting of two matrices of the 12 PPIs for face trials. However, no PPI exhibited significant difference on its own (Table 1).

We next examined what combination of the PPIs, among the 12 PPIs rather than individual PPIs, had the largest difference between retrieval of recent and remote memory by applying MVPA to the multiple PPIs data (Fig. 5, see Materials and Methods). Note that in this MVPA the multiple variables did not in- dicate multiple brain voxels, but did indicate the multiple PPIs. This MVPA revealed that, in both the face and scene trials, the most differential PPI set consisted of three specific PPIs, which enabled us to accurately classify the PPIs into the PPIs in the recent trials or those in the remote trials (for face stimuli: $p=$ 0.003; for scene stimuli: $p=0.00007$; in a permutation test in leave-one-out cross-validation; Fig. $6 A, B$ ). Note that for presentation purposes, results in Table 1 and Figure $6 B$ were based on data from all 43 subjects, respectively. The three PPIs contained a PPI from the perception area to the hippocampal region (PPI for Percep-Hippo), a PPI from the hippocampal region to the posterior temporal region (PPI for Hippo-pTemp), and a PPI from the posterior temporal region to the anterior temporal region (PPI for pTemp-aTemp) (Fig. 6B). In this MVPA using the linear SVM (see Materials and Methods), weight parameters assigned to PPIs in the MVPA enable us to distinguish the PPIs that increased in the remote trials from the PPIs that increased in the recent trials (Dosenbach et al., 2010). Therefore, based on the weight parameters, it was further revealed that, in both of the categories of stimuli, the PPI for Percep-Hippo and pTemp-aTemp in- 
A Paired t-tests of PPI data across subjects to rank PPIs

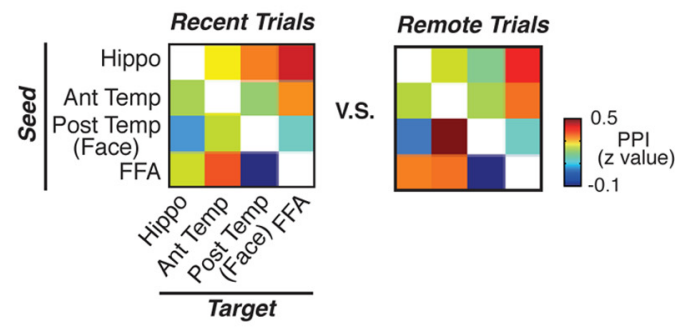

B Building 12 PPI sets based on the ranking of PPIs

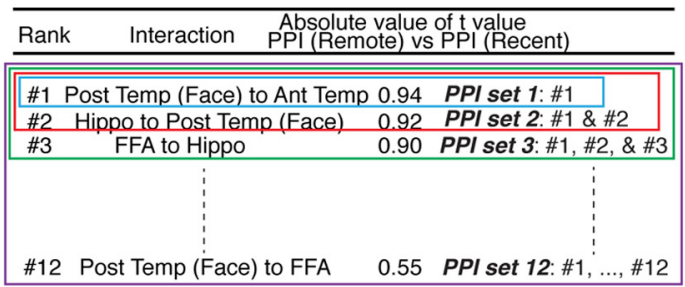

C MVPA using each PPI set

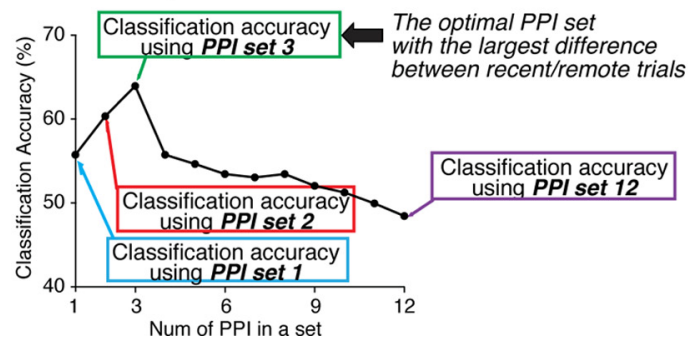

Figure 5. Procedures of MVPA of multiple PPIs. The procedures consist of the following three stages. $A$, We conducted paired $t$ tests of the PPIs across subjects, and ranked all the 12 PPIs in descending order based on the absolute $t$ values in the paired $t$ tests (Table 1). $\boldsymbol{B}$, Based on the ranking table, we sequentially built $12 \mathrm{PPI}$ sets such as PPI sets with only the \#1 PPI, a PPI set with the\#1 and \#2 PPIs, etc. C, Using MVPA, we estimated how accurately each of the 12 PPI sets can be used to classify PPIs into PPIs for the remote trials or those for the recent trials. Consequently, the PPI set with the highest classification accuracy, i.e., PPI set 3, was considered to have the largest difference between the recent and remote trials. The schema shows the analysis procedure for the face trials. We selected the most differential PPI set for the scene trials in the same manner.

creased in the remote trials, whereas the PPI for Hippo-pTemp increased in the recent trials (Fig. $6 \mathrm{C}$ ). To validate the selection, we conducted another type of MVPA by replacing the $t$ test-based ranking (Table 1) with SVM-based ranking, and the same three PPIs were selected as the most differential set of PPIs.

For further characterization of the three PPIs, we estimated the three PPIs based on data in the remember trails and data in the less-remember trials separately (Fig. 6D). In the face trials, the PPI for Percep-Hippo was larger in the remote lessremember trials than in the remote remember trials $(p<0.05$, Bonferroni-corrected in paired $t$ tests). The PPI for HippopTemp was larger in the recent remember trials than in the recent less-remember trials ( $p<0.05$, Bonferroni corrected). The PPI for $\mathrm{pTemp}$-aTemp was larger in the remote remember trials than in the remote less-remember trials $(p<0.05$, Bonferroni corrected). The same dissociation pattern was observed consistently in the scene trials.

The robustness of these results of MVPA applied to PPI was demonstrated in the replications in four different levels across the face and scene trials: (1) the PPI set that was the most differential between the recent and remote trials consisted of the same number of PPIs across the categories of stimuli (i.e., three PPIs; Fig.
$6 A)$; (2) the most differential three PPIs comprised the same set of PPIs (i.e., PPIs for Percep-Hippo, Hippo-pTemp, and pTempaTemp; Fig. $6 \mathrm{~B})$; (3) it was also consistent whether each of the PPIs was increased in the recent trials or in the remote trials (Fig. $6 C)$; and (4) the dissociation pattern of the PPI values between the remember trials and less-remember trials were also replicated across categories (Fig. 6D). This consistency across different categories indicates the robustness of the present results.

\section{Effect of heavy/light repetition of training}

In the abovementioned experiment, to balance the number of the remember trials, the remote stimuli were learned approximately 28 times, while the recent stimuli were learned approximately 3 times. It is possible that the difference of amounts of training repetition influences activations of the four regions (i.e., the hippocampus, anterior temporal region, and posterior temporal regions). To examine the influence, we conducted the additional fMRI experiment using PA tasks, and estimated the difference in the brain activity in these four regions between the remember trials of recent stimuli following heavy repetition of training $(28$ times) and those of recent stimuli following light repetition of training (3 times).

Behaviorally, by focusing on the correct trials with high confidence, the number of the trials was balanced between the correct trials following heavy repetition and those following light repetition (face/scene pairs following heavy repetition of training: $8.3 \pm 0.5 / 8.3 \pm 0.4$; face/scene pairs following light repetition of training: $8.5 \pm 0.6 / 8.4 \pm 0.7$; mean \pm SEM). There was neither main effect $(p>0.6)$ nor interaction $(p>0.5)$ in a repeatedmeasure two-way ANOVA of the response time (face/scene pairs following heavy repetition of training: $0.92 \pm 0.07 \mathrm{~s} / 0.85 \pm$ $0.08 \mathrm{~s}$; face/scene pairs following light repetition of training: $0.89 \pm 0.06 \mathrm{~s} / 0.91 \pm 0.07 \mathrm{~s})$.

To assure the consistency between the main experiment and additional experiment, we first compared the brain activity during the recent trials in the main experiment with that following light training repetition in the additional experiment. As a result, in all the four regions a similar level of the brain activity was observed between the recent trials following light repetition in the additional experiment and the recent trials in the main experiment (Fig. 7), suggesting that the additional experiment had enough power for the ROI analysis.

To estimate the effect of the amounts of training repetition on the brain activity, we then compared the brain activity following the heavy repetition with that following the light repetition. Consequently, no significant effect was observed in any of the four regions: the hippocampal activity during the recent trials following the heavy repetition was not significantly smaller than that following the light repetition $\left(t_{(14)}=-0.35, p>0.63\right.$; Fig. 7$)$. The activity in the anterior and category-specific posterior temporal regions following the heavy repetition was not significantly larger than that following the light repetition (the anterior temporal region: $t_{(14)}=-0.22, p>0.59$; the face-specific posterior temporal region: $t_{(14)}=-0.29, p>0.61$; the scene-specific posterior temporal region: $t_{(14)}=-0.25, p>0.60$ in paired $t$ tests; Figure 7). In an exploratory search for significant effects in a repeatedmeasures two-way ANOVA of the brain activity (heavy/light repetition $\times$ face/scene), neither significant main effects nor interaction was detected in the whole brain at the same threshold as the main experiment ( $p<0.05$, FWE-corrected). These findings suggest that the brain activation found in the main experiment is not related to the different amounts of repetition of training. 
A

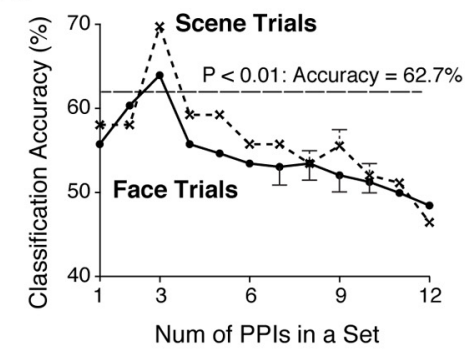

C

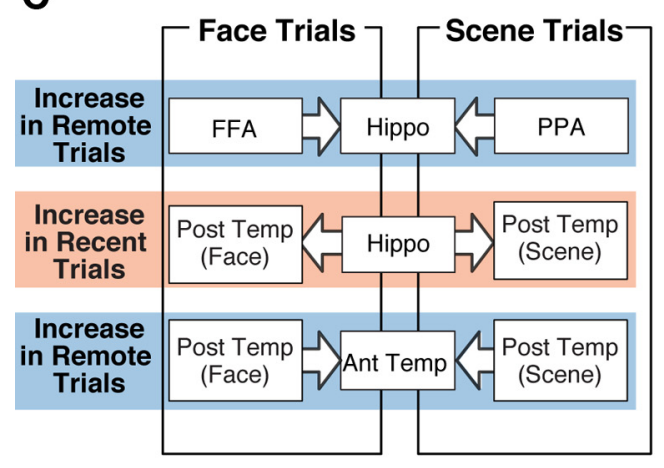

B

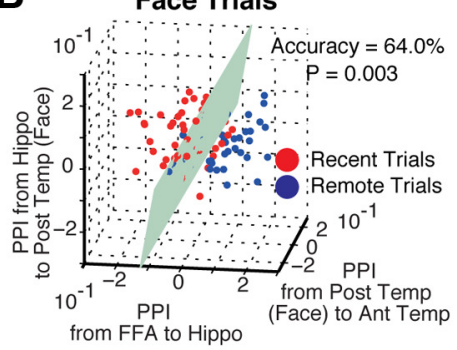

Scene Trials

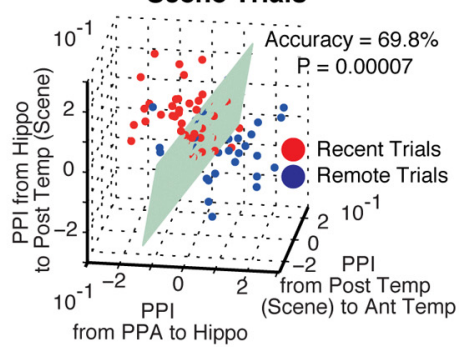

D
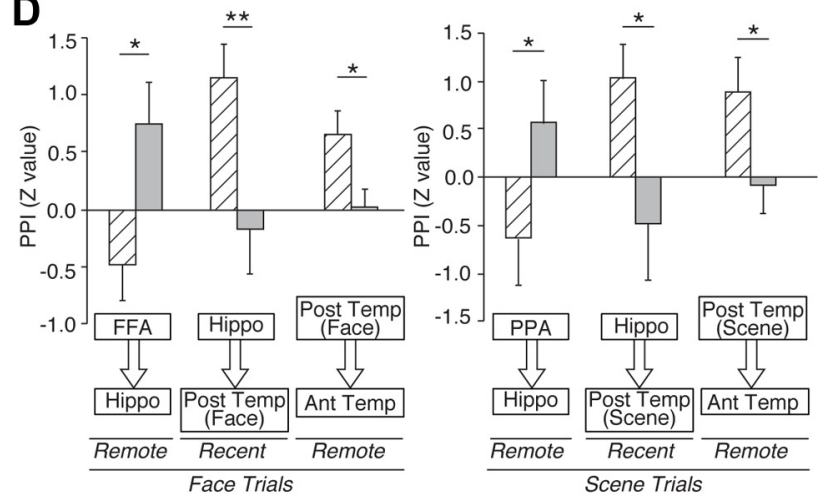

$/ \triangle$ Remember Trials

$\square$ Less-Remember Trials

Figure 6. Results of MVPA of multiple PPIs. A, The most differential PPI sets. In both of the face and scene trials, MVPA using three specific PPIs achieved the highest classification accuracy. The $x$-axis indicates the number of PPIs in a PPI set, and the $y$-axis shows how accurately MVPA could distinguish the data in the recent trials from those in the remote trials. $\boldsymbol{B}$, Two clusters classified by three PPI. Right and left, Show that a single plane can be used to classify the three PPIs into two clusters related to the recent and remote trials. Each of the three axes shows the $z$ values of one of the three PPIs. For presentation purpose, the displayed planes were estimated based on data of all 43 subjects. There are 86 dots ( 43 subjects $\times$ recent/remote trials), each of which represents data in the recent/remote trials of one subject. $C$, Three PPIs in recent and remote trials show whether each of the three PPIs increased in the recent or remote trials. The arrows indicate the directions of the PPI. $\boldsymbol{D}$, Difference in PPI between remember and less-remember trials. The boxes and arrows along the $x$-axis show the three PPIs. The $y$-axis shows the $z$ values of the three PPIs. In both the face and scene trials, only the PPI from the category-specific perception regions to the hippocampal region were lower in the remember trials than in the less-remember trials. ${ }^{*} p<0.05$; ${ }^{* *} p<0.01$ in paired $t$ tests with Bonferroni correction. Error bars indicate SD.
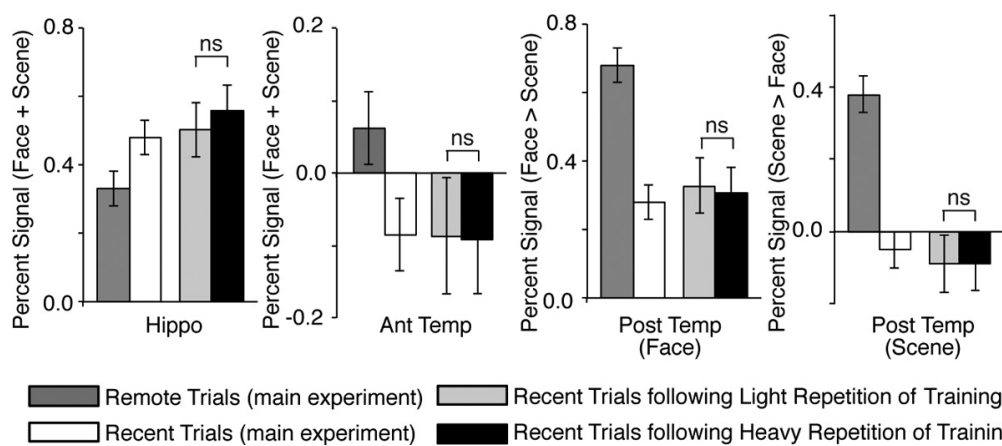

Recent Trials following Light Repetition of Training

Recent Trials following Heavy Repetition of Training

Figure 7. Effect of heavy/light repetition of training. The bars show the brain activity in the four brain regions found in the main experiment (Fig. 2). The bars denoted by the remote and recent trials indicate the brain activity estimated in the main experiment (Fig. 4C), whereas the other bars show the activity estimated in the additional experiment. In all four regions, the brain activity did not show significant difference between the recent trials following the heavy repetition of training and those following the light repetition of training. Error bar indicates SEM.

\section{Discussion}

The present fMRI study provides direct neuroimaging evidence from the brain activations and the behavioral correlations that the posterior temporal cortex is involved in retrieval of remote memory in a category-specific manner, whereas the anterior cortex is involved in retrieval of remote memory in a categorygeneral manner. Furthermore, we also demonstrated the significant interaction from the posterior temporal cortical regions to the anterior temporal cortical region during successful retrieval of remote memory.
To align the retrieval performance between recent and remote memory, we adopted the different training designs with a different number of training cycles between the two types of memory (Fig. $1 B$ ). Consequently, we obtained the sufficient number of remember trials, which enabled us to reveal the clear functional dissociation between the anterior and posterior temporal neocortices. On the other hand, the difference in the training design resulted in a large discrepancy in the number of the training repetitions between the recent and remote memory (i.e., $2.2 \pm 0.7$ vs $27.5 \pm 8.2$ times). Therefore, the different amounts of training repetition may cause a difference in the brain activity in the four brain regions (i.e., the hippocampus, anterior temporal region, and posterior temporal regions). However, the results of the additional experiment suggest that the present activations in the temporal lobe were not significantly influenced by the difference in the training repetition, which is consistent with a previous fMRI study reporting that significant repetition effects were not observed in the temporal lobe (van Turennout et al., 2000). These results suggest that the present brain activations in the anterior and posterior temporal regions are considered to be associated with the difference between recent and remote memory retrieval. As shown previously (Wilson and McNaughton, 1994; 
Carr et al., 2011; McKenzie and Eichenbaum, 2011), the cortical regions would become involved in remote memory through slow-wave sleep (Rasch and Born, 2007; Rasch et al., 2007).

Previous neuropsychological studies have suggested that semantic memory of different categories is stored in different human brain regions (Tranel and Damasio, 1985; Hart and Gordon, 1990; Martin et al., 1995; 1996; Damasio et al., 1996). Even in rats, the different cortical regions are considered to be recruited in different types of remote memory (Ross and Eichenbaum, 2006). In the present study, we found that the STS is related to the remote memory specific to face images, whereas the ITG is related to that specific to scene images (Fig. 4). This dissociation between the dorsal and ventral parts of the temporal neocortex is consistent with previous studies on the neural basis for categoryspecific semantic knowledge (Martin et al., 1995; 1996; Damasio et al., 1996; Chao et al., 1999; Polyn et al., 2005). Previous neuroimaging studies have also shown that the STS is specifically related to semantic knowledge of living things such as famous faces or animals (Chao et al., 1999; Polyn et al., 2005). On the other hand, previous neuropsychological and neuroimaging studies have suggested that the neural representation of nonliving things such as tools and places is located in the more ventral temporal cortex such as the ITG than that of living things (Damasio et al., 1996; Martin et al., 1996; Chao et al., 1999). Our findings provide further validation to this functional dissociation between the dorsal and ventral temporal neocortex.

The present study also investigated the role of the anterior temporal cortical region during remote memory processing. Previous neuropsychological studies have suggested that, compared with the posterior temporal cortex, the anterior temporal cortex is involved in remote memory processing in a more general and integrative way (Mummery et al., 2000; Chan et al., 2001). In a distributed-plus-hub view, the category/modality-specific remote memory is distributed in different brain regions including the posterior temporal cortex, and the anterior temporal cortex plays the role of a hub to gather the distributed memory information (Patterson et al., 2007). Considering previous literature, we can speculate that, during remote memory retrieval, the anterior temporal region gathers memory information for the following high-order processing in a manner irrelevant to specific attributes of the memory. However, it should be noted that these neuropsychological findings (Mummery et al., 2000; Chan et al., 2001; Patterson et al., 2007) are mainly based on observation of patients with impairments in retrieval of semantic knowledge, which is more complicated than arbitrary paired associative memory used in the present study. Therefore, the further studies are required to examine the abovementioned functions of the anterior temporal regions.

The functional interactions revealed in the present study can be interpreted in a manner consistent with the distinct roles of the anterior and posterior temporal cortices. The PPI for HippopTemp was larger in the recent remember trials where the recently learned memory information was considered to be still in the memory transformation process. This result implies that the PPI is involved in the memory transformation of recently encoded pairs from the hippocampus to the posterior temporal cortex, and the memory information is formed in the posterior temporal cortex. This role of the hippocampal-cortical interaction during memory consolidation is consistent with a previous study showing the essential role of the interaction by using genetic modulation in rats (Lesburguères et al., 2011). In addition, the PPI for pTemp-aTemp was increased in the remote remember trials where the subjects were retrieving the memory consol- idated after the repeated learning sessions, suggesting that the anterior temporal region is a convergence point of the information from the posterior temporal regions. This functional interaction from the category-specific posterior temporal regions to the category-general anterior temporal region implies that the anterior temporal region plays the role of a hub to which category-specific remote memory stored in the different posterior temporal regions converges before it is retrieved. This implication is consistent with a recent magneto-encephalography study that revealed that the anteroposterior functional interaction in the temporal neocortex was significantly increased during retrieval of remote memory than during retrieval of recent memory (Nieuwenhuis et al., 2011).

The functional interaction from the category-specific perception region to the hippocampal region can be also interpreted consistently with recent neuroimaging studies (Takashima et al., 2009; Tambini et al., 2010). One of the studies showed that the connectivity between the hippocampus and the perception region increases during resting state after successful memory encoding (Tambini et al., 2010). Another study shows that the interaction decreases after memory consolidation (Takashima et al., 2009). In the present study, the PPI for Percep-Hippo increased in the remote less-remember trials where the subjects were encoding the paired stimuli that they had forgotten and had become unfamiliar with after the long interval of 8 weeks, which may raise the possibility that the PPI reflects the encoding of unfamiliar pairs from the perception areas to the hippocampus. This interpretation is consistent with the results of these previous studies (Takashima et al., 2009; Tambini et al., 2010), and extends the characterization of the hippocampal-neocortical interaction by determining the directionality of the interaction, i.e., from the perception area to the hippocampus.

The present study demonstrated the functional dissociations between the anterior and posterior temporal cortices, and suggests that category-specific remote memory is represented in the posterior temporal cortical regions, and is retrieved commonly through the category-general anterior temporal cortical region. If the anterior temporal region is a convergence point of categoryspecific remote memory, it is rational to assume that the region interacts with other parts of the brain, such as the frontal and parietal lobes. Previous studies suggest that several prefrontal regions play essential roles in the selection of long-term memory (Buckner and Wheeler, 2001; Thompson-Schill, 2003; Frankland and Bontempi, 2005). For instance, the ventromedial prefrontal cortex is proposed to interact with the temporal lobe and facilitate memory consolidation (van Kesteren et al., 2010). Other studies suggest that the parietal regions control neural processes of memory retrieval through attentional modulation (Buckner and Wheeler, 2001; Cabeza et al., 2008; Margulies et al., 2009). Although further studies are needed, the anterior temporal region, therefore, could be one of the target regions with which these frontal and parietal regions interact during the retrieval of remote memory.

\section{References}

Axmacher N, Haupt S, Fernández G, Elger CE, Fell J (2008) The role of sleep in declarative memory consolidation-direct evidence by intracranial EEG. Cereb Cortex 18:500-507.

Bayley PJ, Gold JJ, Hopkins RO, Squire LR (2005) The neuroanatomy of remote memory. Neuron 46:799-810.

Bright P, Moss H, Tyler LK (2004) Unitary vs multiple semantics: PET studies of word and picture processing. Brain Lang 89:417-432.

Buckner RL, Wheeler ME (2001) The cognitive neuroscience of remembering. Nat Rev Neurosci 2:624-634. 
Cabeza R, Ciaramelli E, Olson IR, Moscovitch M (2008) The parietal cortex and episodic memory: an attentional account. Nat Rev Neurosci 9:613-625.

Carr MF, Jadhav SP, Frank LM (2011) Hippocampal replay in the awake state: a potential substrate for memory consolidation and retrieval. Nat Neurosci 14:147-153.

Chan D, Fox NC, Scahill RI, Crum WR, Whitwell JL, Leschziner G, Rossor AM, Stevens JM, Cipolotti L, Rossor MN (2001) Patterns of temporal lobe atrophy in semantic dementia and Alzheimer's disease. Ann Neurol 49:433-442.

Chao LL, Haxby JV, Martin A (1999) Attribute-based neural substrates in temporal cortex for perceiving and knowing about objects. Nat Neurosci 2:913-919.

Damasio H, Grabowski TJ, Tranel D, Hichwa RD, Damasio AR (1996) A neural basis for lexical retrieval. Nature 380:499-505.

Davies RR, Graham KS, Xuereb JH, Williams GB, Hodges JR (2004) The human perirhinal cortex and semantic memory. Eur J Neurosci 20:2441-2446.

De Martino F, Valente G, Staeren N, Ashburner J, Goebel R, Formisano E (2008) Combining multivariate voxel selection and support vector machines for mapping and classification of fMRI spatial patterns. Neuroimage 43:44-58.

Desgranges B, Matuszewski V, Piolino P, Chételat G, Mézenge F, Landeau B, la Sayette de V, Belliard S, Eustache F (2007) Anatomical and functional alterations in semantic dementia: a voxel-based MRI and PET study. Neurobiol. Aging 28:1904-1913.

Devlin JT, Russell RP, Davis MH, Price CJ, Wilson J, Moss HE, Matthews PM, Tyler LK (2000) Susceptibility-induced loss of signal: comparing PET and fMRI on a semantic task. Neuroimage 11:589-600.

Dosenbach NU, Nardos B, Cohen AL, Fair DA, Power JD, Church JA, Nelson SM, Wig GS, Vogel AC, Lessov-Schlaggar CN, Barnes KA, Dubis JW, Feczko E, Coalson RS, JRP Jr, Barch DM, Petersen SE, Schlaggar BL (2010) Prediction of individual brain maturity using fMRI. Science 10:1358-1361.

Edelson M, Sharot T, Dolan RJ, Dudai Y (2011) Following the crowd: brain substrates of long-term memory conformity. Science 333:108-111.

Epstein R, Kanwisher N (1998) A cortical representation of the local visual environment. Nature 392:598-601.

Etkin A, Egner T, Peraza DM, Kandel ER, Hirsch J (2006) Resolving emotional conflict: a role for the rostral anterior cingulate cortex in modulating activity in the amygdala. Neuron 51:871-882.

Frankland PW, Bontempi B (2005) The organization of recent and remote memories. Nat Rev Neurosci 6:119-130.

Friston KJ, Buechel C, Fink GR, Morris J, Rolls E, Dolan RJ (1997) Psychophysiological and modulatory interactions in neuroimaging. Neuroimage 6:218-229.

Gauthier I, Anderson AW, Tarr MJ, Skudlarski P, Gore JC (1997) Levels of categorization in visual recognition studied using functional magnetic resonance imaging. Curr Biol 7:645-651.

Haist F, Bowden Gore J, Mao H (2001) Consolidation of human memory over decades revealed by functional magnetic resonance imaging. Nat Neurosci 4:1139-1145.

Hart J Jr, Gordon B (1990) Delineation of single-word semantic comprehension deficits in aphasia, with anatomical correlation. Ann Neurol $27: 226-231$

Hassabis D, Chu C, Rees G, Weiskopf N, Molyneux PD, Maguire EA (2009) Decoding neuronal ensembles in the human hippocampus. Curr Biol 19:546-554.

Haxby JV, Gobbini MI, Furey ML, Ishai A, Schouten JL, Pietrini P (2001) Distributed and overlapping representations of faces and objects in ventral temporal cortex. Science 293:2425-2430.

Higuchi S, Miyashita Y (1996) Formation of mnemonic neuronal responses to visual paired associates in inferotemporal cortex is impaired by perirhinal and entorhinal lesions. Proc Natl Acad Sci U S A 93:739-743.

Hodges JR, Patterson K, Oxbury S, Funnell E (1992) Semantic dementia. Progressive fluent aphasia with temporal lobe atrophy. Brain 115 (Pt 6):1783-1806.

Inza I, Larrañaga P, Blanco R, Cerrolaza AJ (2004) Filter versus wrapper gene selection approaches in DNA microarray domains. Artif Intell Med 31:91-103.

Kamitani Y, Tong F (2005) Decoding the visual and subjective contents of the human brain. Nat Neurosci 8:679-685.
Kanwisher N, McDermott J, Chun MM (1997) The fusiform face area: a module in human extrastriate cortex specialized for face perception. J Neurosci 17:4302-4311.

Kapur N, Brooks DJ (1999) Temporally-specific retrograde amnesia in two cases of discrete bilateral hippocampal pathology. Hippocampus 9:247-254.

Kapur N, Ellison D, Smith MP, McLellan DL, Burrows EH (1992) Focal retrograde amnesia following bilateral temporal lobe pathology. A neuropsychological and magnetic resonance study. Brain 115 Pt 1:73-85.

Kriegeskorte N, Simmons WK, Bellgowan PS, Baker CI (2009) Circular analysis in systems neuroscience: the dangers of double dipping. Nat Neurosci 12:535-540.

Lesburguères E, Gobbo OL, Alaux-Cantin S, Hambucken A, Trifilieff P, Bontempi B (2011) Early tagging of cortical networks is required for the formation of enduring associative memory. Science 331:924-928.

Manns JR, Hopkins RO, Squire LR (2003) Semantic memory and the human hippocampus. Neuron 38:127-133.

Margulies DS, Vincent JL, Kelly C, Lohmann G, Uddin LQ, Biswal BB, Villringer A, Castellanos FX, Milham MP, Petrides M (2009) Precuneus shares intrinsic functional architecture in humans and monkeys. Proc Natl Acad Sci U S A 106:20069-20074.

Martin A (2007) The representation of object concepts in the brain. Annu Rev Psychol 58:25-45.

Martin A, Chao LL (2001) Semantic memory and the brain: structure and processes. Curr Opin Neurobiol 11:194-201.

Martin A, Haxby JV, Lalonde FM, Wiggs CL, Ungerleider LG (1995) Discrete cortical regions associated with knowledge of color and knowledge of action. Science 270:102-105.

Martin A, Wiggs CL, Ungerleider LG, Haxby JV (1996) Neural correlates of category-specific knowledge. Nature 379:649-652.

McKenzie S, Eichenbaum H (2011) Consolidation and reconsolidation: two lives of memories? Neuron 71:224-233.

Messinger A, Squire LR, Zola SM, Albright TD (2001) Neuronal representations of stimulus associations develop in the temporal lobe during learning. Proc Natl Acad Sci U S A 98:12239-12244

Mitchell TM, Hutchinson R, Niculescu RS, Pereira F, Wang X, Just M, Newman S (2004) Learning to decode cognitive states from brain images. Machine Learning 57:145-175.

Moscovitch M, Nadel L, Winocur G, Gilboa A, Rosenbaum RS (2006) The cognitive neuroscience of remote episodic, semantic and spatial memory. Curr Opin Neurobiol 16:179-190.

Mummery CJ, Patterson K, Price CJ, Ashburner J, Frackowiak RS, Hodges JR (2000) A voxel-based morphometry study of semantic dementia: relationship between temporal lobe atrophy and semantic memory. Ann Neurol 47:36-45

Naya Y, Yoshida M, Miyashita Y (2003) Forward processing of long-term associative memory in monkey inferotemporal cortex. J Neurosci 23:2861-2871.

Nestor PJ, Fryer TD, Hodges JR (2006) Declarative memory impairments in Alzheimer's disease and semantic dementia. Neuroimage 30:1010-1020.

Nieuwenhuis ILC, Takashima A, Oostenveld R, McNaughton BL, Fernández G, Jensen O (2011) The neocortical network representing associative memory reorganizes with time in a process engaging the anterior temporal lobe. Cereb Cortex. Dec. 2.

Patterson K, Nestor PJ, Rogers TT (2007) Where do you know what you know? The representation of semantic knowledge in the human brain. Nat Rev Neurosci 8:976-987.

Polyn SM, Natu VS, Cohen JD, Norman KA (2005) Category-specific cortical activity precedes retrieval during memory search. Science 310:1963-1966.

Rasch B, Born J (2007) Maintaining memories by reactivation. Curr Opin Neurobiol 17:698-703.

Rasch B, Büchel C, Gais S, Born J (2007) Odor cues during slow-wave sleep prompt declarative memory consolidation. Science 315:1426-1429.

Rogers TT, Hocking J, Noppeney U, Mechelli A, Gorno-Tempini ML, Patterson K, Price CJ (2006) Anterior temporal cortex and semantic memory: reconciling findings from neuropsychology and functional imaging. Cogn Affect Behav Neurosci 6:201-213.

Ross RS, Eichenbaum H (2006) Dynamics of hippocampal and cortical activation during consolidation of a nonspatial memory. J Neurosci 26:4852-4859. 
Ruiz R, Riquelme JC, Aguilar-Ruiz JS (2006) Incremental wrapper-based gene selection from microarray data for cancer classification. Pattern Recogn 39:2383-2392.

Saeys Y, Inza I, Larrañaga P (2007) A review of feature selection techniques in bioinformatics. Bioinformatics 23:2507-2517.

Sakai K, Miyashita Y (1991) Neural organization for the long-term memory of paired associates. Nature 354:152-155.

Scoville WB, Milner B (1957) Loss of recent memory after bilateral hippocampal lesions. J Neurol Neurosurg Psychiatr 20:11-21.

Squire LR, Bayley PJ (2007) The neuroscience of remote memory. Curr Opin Neurobiol 17:185-196.

Stark CE, Squire LR (2000) fMRI activity in the medial temporal lobe during recognition memory as a function of study-test interval. Hippocampus 10:329-337.

Stephan KE, Friston KJ (2010) Analyzing effective connectivity with functional magnetic resonance imaging. WIREs Cogni Sci 1:446-459.

Takashima A, Petersson KM, Rutters F, Tendolkar I, Jensen O, Zwarts MJ, McNaughton BL, Fernández G (2006) Declarative memory consolidation in humans: a prospective functional magnetic resonance imaging study. Proc Natl Acad Sci U S A 103:756-761.

Takashima A, Nieuwenhuis IL, Jensen O, Talamini LM, Rijpkema M, Fernández G (2009) Shift from hippocampal to neocortical centered retrieval network with consolidation. J Neurosci 29:10087-10093.

Tambini A, Ketz N, Davachi L (2010) Enhanced brain correlations during rest are related to memory for recent experiences. Neuron 65:280-290.

Thompson-Schill SL (2003) Neuroimaging studies of semantic memory: inferring "how" from "where." Neuropsychologia 41:280-292.
Tranel D, Damasio AR (1985) Knowledge without awareness: an autonomic index of facial recognition by prosopagnosics. Science 228:1453-1454.

Tzourio-Mazoyer N, Landeau B, Papathanassiou D, Crivello F, Etard O, Delcroix N, Mazoyer B, Joliot M (2002) Automated anatomical labeling of activations in SPM using a macroscopic anatomical parcellation of the MNI MRI single-subject brain. Neuroimage 15:273-289.

van Kesteren MT, Fernández G, Norris DG, Hermans EJ (2010) Persistent schema-dependent hippocampal-neocortical connectivity during memory encoding and postencoding rest in humans. Proc Natl Acad Sci U S A 107:7550-7555.

van Turennout M, Ellmore T, Martin A (2000) Long-lasting cortical plasticity in the object naming system. Nat Neurosci 3:1329-1334.

Warrington EK (1975) The selective impairment of semantic memory. Q J Exp Psychol 27:635-657.

Watanabe T, Hirose S, Wada H, Katsura M, Chikazoe J, Jimura K, Imai Y, Machida T, Shirouzu I, Miyashita Y, Konishi S (2011) Prediction of subsequent recognition performance using brain activity in the medial temporal lobe. Neuroimage 54:3085-3092.

Wilson MA, McNaughton BL (1994) Reactivation of hippocampal ensemble memories during sleep. Science 265:676-679.

Xiong M, Fang X, Zhao J (2001) Biomarker identification by feature wrappers. Genome Res 11:1878-1887.

Yamashita K, Hirose S, Kunimatsu A, Aoki S, Chikazoe J, Jimura K, Masutani Y, Abe O, Ohtomo K, Miyashita Y, Konishi S (2009) Formation of longterm memory representation in human temporal cortex related to pictorial paired associates. J Neurosci 29:10335-10340.

Zola-Morgan SM, Squire LR (1990) The primate hippocampal formation: evidence for a time-limited role in memory storage. Science 250:288 -290. 